\title{
Multiple solutions of semilinear degenerate elliptic boundary value prob- lems II
}

\section{Kazuaki Taira ${ }^{1 *}$}

${ }^{1}$ Institute of Mathematics, University of Tsukuba, Tsukuba 305-8571, Japan

Received December 2008

Key words Semilinear elliptic boundary value problem, degenerate boundary condition, Leray-Schauder degree, Schauder's fixed point theorem MSC (2000) 35J65, 35J25, 47H10

Dedicated to Professor Izumi Kubo on the occasion of his 70th birthday

The purpose of this paper is to study a class of semilinear degenerate elliptic boundary value problems depending on a parameter which include as particular cases the Dirichlet problem and the Robin problem. By using Schauder's fixed point theorem and the Leray-Schauder degree, we derive lower bounds on the number of solutions of our problem. The results here extend earlier theorems due to Kazdan-Warner and also Amann-Hess to the degenerate case.

Copyright line will be provided by the publisher

\section{Statement of main results}

Let $\Omega$ be a bounded domain of Euclidean space $\mathbf{R}^{N}, N \geq 2$, with smooth boundary $\partial \Omega$; its closure $\bar{\Omega}=\Omega \cup \partial \Omega$ is an $N$ dimensional, compact smooth manifold with boundary. Let $A$ be a second-order, elliptic differential operator with real coefficients such that

$$
A u:=-\sum_{i=1}^{N} \frac{\partial}{\partial x_{i}}\left(\sum_{j=1}^{N} a^{i j}(x) \frac{\partial u}{\partial x_{j}}\right)+c(x) u .
$$

Here:

(1) $a^{i j} \in C^{\infty}(\bar{\Omega})$ and $a^{i j}(x)=a^{j i}(x)$ on $\bar{\Omega}$, and there exists a positive constant $a_{0}$ such that

$$
\sum_{i, j=1}^{N} a^{i j}(x) \xi_{i} \xi_{j} \geq a_{0}|\xi|^{2} \quad \text { for all }(x, \xi) \in \bar{\Omega} \times \mathbf{R}^{N} .
$$

(2) The function $c(x)$ is real-valued and may be discontinuous in $\Omega$. More precisely, $c \in L^{\infty}(\Omega)$ and $c(x) \geq 0$ almost everywhere in $\Omega$.

Let $B$ be a first-order, boundary condition with real coefficients such that

$$
B u:=a\left(x^{\prime}\right) \frac{\partial u}{\partial \nu}+b\left(x^{\prime}\right) u .
$$

Here:

(3) $a \in C^{\infty}(\partial \Omega)$ and $a\left(x^{\prime}\right) \geq 0$ on $\partial \Omega$.

(4) $b \in C^{\infty}(\partial \Omega)$ and $b\left(x^{\prime}\right) \geq 0$ on $\partial \Omega$.

\footnotetext{
* E-mail: taira@math.tsukuba.ac.jp
} 
(5) $\partial / \partial \boldsymbol{\nu}$ is the (exterior) conormal derivative associated with the operator $A$.

Our fundamental hypotheses on the boundary condition $B$ are the following:

(H.1) $a\left(x^{\prime}\right)+b\left(x^{\prime}\right)>0$ on $\partial \Omega$.

(H.2) $b\left(x^{\prime}\right) \not \equiv 0$ on $\partial \Omega$.

It should be emphasized that the boundary condition $B$ is degenerate from an analytical point of view. This is due to the fact that the so-called Lopatinskii-Shapiro complementary condition is violated at each point of the set $M=\left\{x^{\prime} \in \partial \Omega: a\left(x^{\prime}\right)=0\right\}$ (cf. [7]). More precisely, it is easy to verify that the boundary condition $B$ is non-degenerate if and only if either $a\left(x^{\prime}\right)>0$ on $\partial \Omega$ (the Robin case) or $a\left(x^{\prime}\right) \equiv 0$ and $b\left(x^{\prime}\right)>0$ on $\partial \Omega$ (the Dirichlet case).

The purpose of this paper is to study the following semilinear elliptic boundary value problem depending on a parameter: Given a function $f(x, \xi, t)$ defined on $\bar{\Omega} \times \mathbf{R} \times \mathbf{R}$, find a function $u(x)$ in $\Omega$ such that

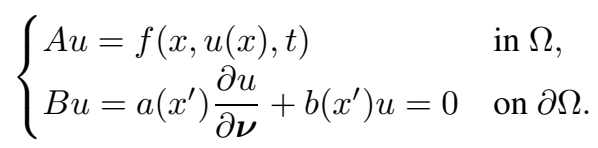

In order to study problem (1.3) in the framework of Sobolev spaces of $L^{p}$ type, we consider the linear elliptic boundary value problem

$$
\begin{cases}A u=g & \text { in } \Omega, \\ B u=0 & \text { on } \partial \Omega\end{cases}
$$

in the framework of the Hilbert space $L^{2}(\Omega)$. We associate with problem (1.4) a densely defined, closed linear operator

$$
\mathfrak{A}: L^{2}(\Omega) \longrightarrow L^{2}(\Omega)
$$

as follows:

(1) $\mathcal{D}(\mathfrak{A})=\left\{u \in W^{2,2}(\Omega): B u=0\right.$ on $\left.\partial \Omega\right\}$.

(2) $\mathfrak{A} u=A u$ for all $u \in \mathcal{D}(\mathfrak{A})$.

Here and in the following the Sobolev space $W^{k, p}(\Omega)$ for $k \in \mathbf{N}$ and $1<p<\infty$ is defined as follows:

$$
\begin{aligned}
W^{k, p}(\Omega)= & \text { the space of functions } u \in L^{p}(\Omega) \text { whose derivatives } D^{\alpha} u, \\
& |\alpha| \leq k, \text { in the sense of distributions are in } L^{p}(\Omega),
\end{aligned}
$$

and its norm $\|\cdot\|_{W^{k, p}(\Omega)}$ is given by the formula

$$
\|u\|_{W^{k, p}(\Omega)}=\left(\sum_{|\alpha| \leq k} \int_{\Omega}\left|D^{\alpha} u(x)\right|^{p} d x\right)^{1 / p} .
$$

Then we have the following fundamental spectral results (i), (ii) and (iii) of the operator $\mathfrak{A}$ (see [13, Theorem 5.1]):

(i) The operator $\mathfrak{A}$ is positive and selfadjoint in $L^{2}(\Omega)$.

(ii) The first eigenvalue $\lambda_{1}$ of $\mathfrak{A}$ is positive and algebraically simple, and its corresponding eigenfunction $\varphi_{1} \in$ $W^{2, p}(\Omega)$, with $N<p<\infty$, may be chosen to be strictly positive in $\Omega$. Namely, we have the assertions

$$
\begin{cases}A \varphi_{1}=\lambda_{1} \varphi_{1} & \text { in } \Omega, \\ \varphi_{1}>0 & \text { in } \Omega, \\ B \varphi_{1}=0 & \text { on } \partial \Omega .\end{cases}
$$


(iii) No other eigenvalues $\lambda_{j}, j \geq 2$, have positive eigenfunctions.

Now we impose the following conditions (F.1) through (F.4) on the nonlinear term $f(x, \xi, t)$ :

(F.1) The function $f(x, \xi, t)$ is real-valued and continuous on $\bar{\Omega} \times \mathbf{R} \times \mathbf{R}$, and its partial derivatives $\partial f / \partial \xi$ and $\partial f / \partial t$ exist and are continuous on $\bar{\Omega} \times \mathbf{R} \times \mathbf{R}$.

(F.2) For every $m \in \mathbf{R}$, there exists a continuous function $h_{m}(x)$ on $\bar{\Omega}$, depending on $m$, such that we have, for all $x \in \Omega, \xi \geq m$ and $t \in \mathbf{R}$,

$$
\frac{\partial f}{\partial t}(x, \xi, t) \geq h_{m}(x)>0 \text {. }
$$

(F.3a) For every $x \in \bar{\Omega}$ and $t \in \mathbf{R}$, we have the assertion

$$
\limsup _{\xi \rightarrow-\infty} \frac{f(x, \xi, t)}{\xi}<\lambda_{1}
$$

where $\lim \sup _{\xi \rightarrow-\infty}$ is assumed to be uniform for $x \in \bar{\Omega}$ and for $t$ in any bounded interval of $\mathbf{R}$.

(F.3b) For every $x \in \bar{\Omega}$ and $t \in \mathbf{R}$, we have the assertion

$$
\liminf _{\xi \rightarrow+\infty} \frac{f(x, \xi, t)}{\xi}>\lambda_{1}
$$

where $\liminf \operatorname{in}_{\xi \rightarrow+\infty}$ is assumed to be uniform for $x \in \bar{\Omega}$ and for $t$ in any bounded interval of $\mathbf{R}$.

(F.4) For every $x \in \bar{\Omega}$ and $t \in \mathbf{R}$, we have the assertion

$$
\limsup _{\xi \rightarrow+\infty} \frac{f(x, \xi, t)}{\xi}<+\infty,
$$

where $\lim \sup _{\xi \rightarrow+\infty}$ is assumed to be uniform for $x \in \bar{\Omega}$ and for $t$ in any bounded interval of $\mathbf{R}$.

A function $u \in W^{2, p}(\Omega)$, with $N<p<\infty$, is called a solution of problem (1.3) if it satisfies the conditions

$$
\begin{cases}A u=f(x, u(x), t) & \text { almost everywhere in } \Omega, \\ B u=0 & \text { on } \partial \Omega .\end{cases}
$$

Here it should be noticed that we have, by Sobolev's imbedding theorem (see [1, Theorem 4.12]),

$$
W^{2, p}(\Omega) \subset C^{2-N / p}(\bar{\Omega}) \subset C^{1}(\bar{\Omega})
$$

if $N<p<\infty$.

We remark that Amann [2] and Amann-Hess [3, Theorem] studied problem (1.3) under the condition that the boundary $\partial \Omega$ is the disjoint union of the two closed subsets $M=\left\{x^{\prime} \in \partial \Omega: a\left(x^{\prime}\right)=0\right\}$ and $\partial \Omega \backslash M=\left\{x^{\prime} \in\right.$ $\left.\partial \Omega: a\left(x^{\prime}\right)>0\right\}$, each of which is an $(N-1)$ dimensional compact smooth manifold.

Our approach to problem (1.3) is based on the Leray-Schauder topological degree. The crucial point is how to control the discontinuous function $c(x)$ in formula (1.1) in terms of Sobolev spaces of $L^{p}$ type, just as in Taira [13].

The main purpose of this paper is to derive lower bounds on the number of solutions of problem (1.3). The next theorem extends Kazdan-Warner [8, Corollary 3.11] and also Amann-Hess [3, Theorem] to the degenerate case (cf. [12, Theorem 6.5.3/1]):

Theorem 1.1 Assume that the nonlinear term $f(x, \xi, t)$ satisfies conditions (F.1) through (F.4). Then there exists a number $t_{0} \in \mathbf{R}$ such that problem (1.3) has no solution for $t>t_{0}$, at least one solution for $t=t_{0}$, and at least two distinct solutions for $t<t_{0}$. 
In the previous paper [14], we proved that conditions (F.1) through (F.3) (without the uniformity assumption with respect to $t$ ) imply the existence of a number $t_{0} \in \mathbf{R}$ such that problem (1.3) has no solution for $t>t_{0}$ and at least one solution for $t<t_{0}$. However, no multiplicity result is obtained there and no assertion is made for $t=t_{0}$ (see [14, Theorem 1.5]).

The following diagram gives a bird's eye view of conditions in the present paper and the previous paper [14], and how these relate to each other:

\begin{tabular}{|c|c|c|}
\hline $\begin{array}{c}\text { Conditions in the } \\
\text { present paper }\end{array}$ & $\begin{array}{c}\text { Conditions in the } \\
\text { previous paper [14] }\end{array}$ & $\begin{array}{c}\text { Conditions in the } \\
\text { previous paper [14] }\end{array}$ \\
\hline (F.2) & (D.1) & $\Longrightarrow(4.1)$ \\
\hline (F.3a) & (D.2) & $\Longrightarrow\left(B_{-}\right) \Longrightarrow\left(A_{-}\right)$ \\
\hline (F.3b) & (b.2) & $\Longrightarrow$ (D.3) \\
\hline
\end{tabular}

The crucial point in the proof of Theorem 1.1 is how to find the second solution of problem $(1.3)$ for $t<t_{0}$, by using Schauder's fixed point theorem and the Leray-Schauder fixed point index. The Leray-Schauder topological degree is an important tool introduced by Leray and Schauder [9] in the study of nonlinear partial differential equations (cf. [10]). The non-triviality of the degree guarantees the existence of a solution of nonlinear equations.

Furthermore, the next corollary is a generalization of Kazdan-Warner [8, Corollary 3.12] to the degenerate case (see also [4, Theorem 3.1]; [5, Theorem 1]; [12, Theorem 6.5.3/3]):

Corollary 1.2 Let the nonlinear term $f(x, \xi, t)$ be of the special form

$$
f(x, \xi, t)=f_{0}(x)+\operatorname{tr}(x)+g(x, \xi), \quad(x, \xi, t) \in \bar{\Omega} \times \mathbf{R} \times \mathbf{R},
$$

where $f_{0} \in C(\bar{\Omega}), r \in C(\bar{\Omega})$ with $r(x)>0$ in $\Omega$ and $g \in C^{1}(\bar{\Omega} \times \mathbf{R})$. We assume that the function $g(x, \xi)$ satisfies the conditions

$$
\begin{aligned}
& \limsup _{\xi \rightarrow-\infty} \frac{g(x, \xi)}{\xi}<\lambda_{1}, \\
& \lambda_{1}<\liminf _{\xi \rightarrow+\infty} \frac{g(x, \xi)}{\xi} \leq \limsup _{\xi \rightarrow+\infty} \frac{g(x, \xi)}{\xi}<+\infty,
\end{aligned}
$$

where $\limsup _{\xi \rightarrow \pm \infty}$ and $\lim _{\inf _{\xi \rightarrow+\infty}}$ are assumed to be uniform for $x \in \bar{\Omega}$. Then there exists a number $t_{0} \in \mathbf{R}$ such that the semilinear elliptic boundary value problem

$$
\begin{cases}A u=f_{0}(x)+\operatorname{tr}(x)+g(x, u(x)) & \text { in } \Omega, \\ B u=0 & \text { on } \partial \Omega\end{cases}
$$

has no solution for $t>t_{0}$, at least one solution for $t=t_{0}$, and at least two solutions for $t<t_{0}$.

Corollary 1.2 follows by verifying all the conditions (F.1) through (F.4) of Theorem 1.1 for the nonlinear term

$$
f(x, \xi, t)=f_{0}(x)+\operatorname{tr}(x)+g(x, \xi) \text { for all }(x, \xi, t) \in \bar{\Omega} \times \mathbf{R} \times \mathbf{R} .
$$

In the previous paper [14], we proved that problem (1.5) has no solution for $t>t_{0}$ and at least one solution for $t<t_{0}$, while no assertion is made for $t=t_{0}$ (see [14, Corollary 1.7]).

We can interpret Corollary 1.2 as follows (cf. [5]): Given two functions $f(x)$ and $g(x, \xi)$ defined in $\Omega$ and on $\bar{\Omega} \times \mathbf{R}$, respectively, we consider the following semilinear elliptic boundary value problem

$$
\begin{cases}A u=f(x)+g(x, u(x)) & \text { in } \Omega \\ B u=0 & \text { on } \partial \Omega .\end{cases}
$$

Given a positive smooth function $r(x)$ in $\Omega$, we decompose the function $f(x)$ as an orthogonal sum in the Hilbert space $L^{2}(\Omega)$ (see [14, decomposition (3.4)])

$$
f(x)=f_{0}(x)+t r(x), \quad t \in \mathbf{R},
$$


and regard the nonlinear term $g(x, u(x))$ as fixed. In this setting, Corollary 1.2 asserts that there exists a number $t_{0}=t_{0}\left(f_{0}, r, g\right) \in \mathbf{R}$, depending on $f_{0}, r$ and $g$, such that problem (1.6) has no solution for $t>t_{0}$, at least one solution for $t=t_{0}$, and at least two solutions for $t<t_{0}$.

The rest of this paper is organized as follows. In Section 2 we prove Theorem 1.1 by using degree-theoretic arguments, just as in Amann-Hess [3, Theorem]. However, it should be emphasized that our proof of Theorem 1.1 is based on the previous work [13] and [14]. In [13] we have developed a new KreĬn-Rutman theory for a class of degenerate elliptic eigenvalue problems with indefinite weights that model population dynamics in environments with spatial heterogeneity ( [13, Section 2]). In [14] we have proved how the linear theory may be used to obtain monotone methods which lead to existence theorems for a class of semilinear elliptic boundary value problems with degenerate boundary conditions ( [14, Theorem 1.3]). The crucial point in the proof of Theorem 1.1 is how to find the second solution of problem (1.3) for $t<t_{0}$. To do this, we make use of Schauder's fixed point theorem (Theorem 2.5) and Kronecker's existence theorem for the Leray-Schauder degree (Theorem 2.8).

\section{Proof of Theorem 1.1}

This section is devoted to the proof of Theorem 1.1 which is inspired by Amann-Hess [3, Theorem]. The crucial point in the proof is how to find the second solution of problem (1.3) for $t<t_{0}$, by using Schauder's fixed point theorem and the Leray-Schauder fixed point index. The proof of Theorem 1.1 is divided into eight steps.

Step 1: First, we remark that conditions (F.2), (F.3a) and (F.3b) imply that conditions (D.1), (D.2) and (D.3) of [14, Theorem 1.5] are satisfied. Hence, by applying [14, Theorem 1.5] to our situation we can find a number $t_{0} \in \mathbf{R}$ such that problem (1.3) has no solution for $t>t_{0}$ and at least one solution for $t<t_{0}$.

We take a number $t^{*}<t_{0}$ and let $\tau \in\left(t^{*}, t_{0}\right)$. Then there exists a function $\bar{u} \in W^{2, p}(\Omega)$, with $N<p<\infty$, such that

$$
\begin{cases}A \bar{u}=f(x, \bar{u}(x), \tau) & \text { in } \Omega, \\ B \bar{u}=0 & \text { on } \partial \Omega .\end{cases}
$$

Moreover, we have the following:

Lemma 2.1 The function $\bar{u} \in W^{2, p}(\Omega)$, with $N<p<\infty$, is a strict supersolution of problem (1.3) for $t=t^{*}$. Namely, $\bar{u}$ is a supersolution of problem (1.3) for $t=t^{*}$, but is not a solution of problem (1.3) for $t=t^{*}$.

Proof. By condition (F.2), it follows that the function $f(x, \xi, t)$ is strictly increasing in the variable $t$. Hence we have, for $t^{*}<\tau<t_{0}$,

$$
\begin{cases}A \bar{u}=f(x, \bar{u}(x), \tau)>f\left(x, \bar{u}(x), t^{*}\right) & \text { in } \Omega, \\ B \bar{u}=0 & \text { on } \partial \Omega .\end{cases}
$$

This proves that function $\bar{u}(x)$ is a strict supersolution of problem (1.3) for $t=t^{*}$.

On the other hand, we remark that conditions (F.2) and (F.3a) imply that conditions (4.1) and $\left(A_{-}\right)$of [14, Theorem 4.2] are satisfied. Hence, by arguing just as in the proof of [14, Theorem 4.2] we can construct a strict subsolution $\underline{u} \in W^{2, p}(\Omega)$, with $N<p<\infty$, of problem (1.3) for $t=t^{*}$ which satisfies the conditions

$$
\begin{cases}A \underline{u}<f\left(x, \underline{u}(x), t^{*}\right) & \text { in } \Omega, \\ \underline{u}(x)<\bar{u}(x) & \text { in } \Omega, \\ B \underline{u}=0 & \text { on } \partial \Omega .\end{cases}
$$

Here the notation $u<v$ means that $u(x) \leq v(x)$ in $\Omega$ but $u(x) \not \equiv v(x)$ in $\Omega$.

Step 2: We let

$$
\omega_{0}:=\max \left\{\left|\frac{\partial f}{\partial \xi}\left(x, \xi, t^{*}\right)\right|: x \in \bar{\Omega}, \min _{\bar{\Omega}} \underline{u} \leq \xi \leq \max _{\bar{\Omega}} \bar{u}\right\},
$$


and

$$
\omega:=\max \left\{\omega_{0}+1,\|c\|_{L^{\infty}(\Omega)}\right\} .
$$

We modify the nonlinear term $f(x, \xi, t)$ as follows:

$$
f_{\omega}(x, \xi, t):=f(x, \xi, t)+\omega \xi \text { for all }(x, \xi, t) \in \bar{\Omega} \times \mathbf{R} \times \mathbf{R} .
$$

Then, by applying [13, Theorem 1.1] with $c(x):=c(x)+\omega$ and $\varphi:=0$ we obtain that the linear boundary value problem

$$
\begin{cases}(A+\omega) u=v & \text { in } \Omega \\ B u=0 & \text { on } \partial \Omega\end{cases}
$$

has a unique solution $u \in W^{2, p}(\Omega)$ for any $v \in L^{p}(\Omega)$ with $N<p<\infty$. Therefore, we can introduce a continuous linear operator (resolvent)

$$
K_{\omega}: L^{p}(\Omega) \longrightarrow W^{2, p}(\Omega)
$$

by the formula $u=K_{\omega} v$. Moreover, by the Ascoli-Arzelà theorem it follows that the resolvent $K_{\omega}$, considered as an operator

$$
K_{\omega}: C(\bar{\Omega}) \longrightarrow C^{1}(\bar{\Omega})
$$

is compact if $N<p<\infty$. Indeed, it suffices to note (see [1, Theorem 4.12]) that the Sobolev space $W^{2, p}(\Omega)$ is continuously imbedded into the Hölder space $C^{2-N / p}(\bar{\Omega})$ with $2-N / p>1$, for all $N<p<\infty$.

Finally, we let

$$
E:=C_{B}^{1}(\bar{\Omega})=\left\{u \in C^{1}(\bar{\Omega}): B u=0 \quad \text { on } \partial \Omega\right\}
$$

be an ordered Banach space equipped with the norm of $C^{1}(\bar{\Omega})$ and with the natural ordering $\leq$. We remark that the positive cone $P$ is given by the formula

$$
P:=\left\{u \in C_{B}^{1}(\bar{\Omega}): u(x) \geq 0 \text { on } \bar{\Omega}\right\} .
$$

Now it is easy to see that problem (1.3) is equivalent to the fixed point equation

$$
u=K_{\omega}\left(F_{\omega}(u, t)\right) \quad \text { in } E
$$

where $F_{\omega}$ is the Nemytskii operator of the nonlinear term $f_{\omega}$ defined by the formula

$$
F_{\omega}(u, t):=f_{\omega}(x, u(x), t)=f(x, u(x), t)+\omega u(x) \quad \text { for all }(x, t) \in \bar{\Omega} \times \mathbf{R} .
$$

Indeed, it suffices to note that

$$
\begin{aligned}
& \left\{\begin{array} { l l } 
{ A u = f ( x , u ( x ) , t ) } & { \text { in } \Omega , } \\
{ B u = 0 } & { \text { on } \partial \Omega }
\end{array} \Longleftrightarrow \left\{\begin{array}{ll}
(A+\omega) u=f_{\omega}(x, u(x), t) & \text { in } \Omega, \\
B u=0 & \text { on } \partial \Omega
\end{array}\right.\right. \\
& \Longleftrightarrow u=K_{\omega}\left(F_{\omega}(u, t)\right) \quad \text { in } E \text {. }
\end{aligned}
$$

Step 3: We are reduced to the study of the fixed point equation (2.2). First, we have the following:

Lemma 2.2 The mapping

$$
K_{\omega} F_{\omega}: E \times \mathbf{R} \longrightarrow E
$$

is continuous, and maps bounded sets into relatively compact sets. 
Proof. Let $\left\{\left(u_{n}, t_{n}\right)\right\}$ be an arbitrary bounded sequence in $E \times \mathbf{R}$. Then, since $F_{\omega}: E \times \mathbf{R} \rightarrow C(\bar{\Omega})$ is bounded and since $K_{\omega}: C(\bar{\Omega}) \rightarrow E$ is compact, we can find a subsequence $\left\{\left(u_{n^{\prime}}, t_{n^{\prime}}\right)\right\}$ such that the sequence $\left\{K_{\omega}\left(F_{\omega}\left(u_{n^{\prime}}, t_{n^{\prime}}\right)\right)\right\}$ is convergent in $E$.

Secondly, we have the following:

Lemma 2.3 The mapping $K_{\omega} F_{\omega}\left(\cdot, t^{*}\right)$ is strongly increasing on the order interval

$$
X:=\{u \in E: \underline{u} \leq u \leq \bar{u}\} .
$$

Namely, if $u<v$ in $X$, then it follows that $K_{\omega}\left(F_{\omega}\left(v, t^{*}\right)\right)-K_{\omega}\left(F_{\omega}\left(u, t^{*}\right)\right)$ belongs to the interior $\operatorname{Int}(P)$ of the positive cone $P$.

Pro o f. By applying [14, Lemmas 2.1 and 2.2] to the mapping $K_{\omega} F_{\omega}\left(\cdot, t^{*}\right)$, we obtain that $K_{\omega} F_{\omega}\left(\cdot, t^{*}\right)$ maps $X$ into itself and is increasing on $X$.

It remains to show that $K_{\omega} F_{\omega}\left(\cdot, t^{*}\right)$ is strongly increasing on $X$. If $u<v$ in $X$, we let

$$
w:=K_{\omega}\left(F_{\omega}\left(v, t^{*}\right)\right)-K_{\omega}\left(F_{\omega}\left(u, t^{*}\right)\right) \in W^{2, p}(\Omega), \quad N<p<\infty .
$$

Then it follows from the definition (2.1) of $\omega$ that

$$
\begin{aligned}
(A+\omega) w & =f_{\omega}\left(x, v(x), t^{*}\right)-f_{\omega}\left(x, u(x), t^{*}\right) \\
& =f\left(x, v(x), t^{*}\right)-f\left(x, u(x), t^{*}\right)+\omega(v(x)-u(x)) \\
& >0 \quad \text { in } \Omega,
\end{aligned}
$$

and that

$$
B w=0 \quad \text { on } \partial \Omega .
$$

Therefore, by exploiting the maximum principle in its full length we obtain from [13, Proposition 3.12] that

$$
\begin{cases}w=0 & \text { on } M=\left\{x^{\prime} \in \partial \Omega: a\left(x^{\prime}\right)=0\right\} \\ \frac{\partial w}{\partial \nu}<0 & \text { on } M \\ w>0 & \text { on } \bar{\Omega} \backslash M=\Omega \cup(\partial \Omega \backslash M) .\end{cases}
$$

By assertion (2.3), it is easy to see that $w \in \operatorname{Int}(P)$.

The proof of Lemma 2.3 is complete.

Thirdly, we have the following:

Lemma 2.4 The mapping $K_{\omega} F_{\omega}\left(\cdot, t^{*}\right): X=[\underline{u}, \bar{u}] \rightarrow E$ is compact, that is, the closure of the image $K_{\omega} F_{\omega}\left(X, t^{*}\right)$ is compact in $E$.

Proof. We have only to show that the image $K_{\omega} F_{\omega}\left(X, t^{*}\right)$ is sequentially compact in $E$. Let $\left\{v_{n}\right\}$ be an arbitrary sequence in $K_{\omega} F_{\omega}\left(X, t^{*}\right)$ with

$$
v_{n}=K_{\omega}\left(F_{\omega}\left(u_{n}, t^{*}\right)\right)
$$

for some $u_{n} \in X$. Then, since the order interval $X$ is bounded in $C(\bar{\Omega})$ and since $K_{\omega}: C(\bar{\Omega}) \rightarrow E$ is compact, we can find a subsequence $\left\{u_{n^{\prime}}\right\}$ in $X$ such that $\left\{K_{\omega}\left(F_{\omega}\left(u_{n^{\prime}}, t^{*}\right)\right)\right\}$ is convergent in $E$.

Step 4: We let

$$
G:=K_{\omega} F_{\omega}\left(\cdot, t^{*}\right),
$$

and show that the mapping $G$ has at least two distinct fixed points.

To do this, we need an extension of Brouwer's fixed point theorem to the infinite-dimensional case, due to Schauder (see [6, Theorem 3.4.7]; [11, Theorem 2.1.2]; [15, Theorem 2.A]): 
Theorem 2.5 Let $C$ be a non-empty closed, bounded and convex subset of a real Banach space E. A compact mapping $f: C \rightarrow C$ has a fixed point $x$ in $C: f(x)=x$.

We remark that the order interval $X=[\underline{u}, \bar{u}]$ is closed, bounded and convex in the Banach space $C(\bar{\Omega})$. Moreover, it follows from Lemmas 2.3 and 2.4 that $G(X) \subset X$ and that the closure $\overline{G(X)}$ is compact in $C(\bar{\Omega})$. Therefore, by applying Schauder's fixed point theorem (Theorem 2.5) with $E:=C(\bar{\Omega}), C:=X$ and $f:=G$ we obtain that $G$ has a fixed point $u_{0}$ in $X$ :

$$
G\left(u_{0}\right)=u_{0}, \quad u_{0} \in X=[\underline{u}, \bar{u}] .
$$

Without loss of generality, we may assume that $u_{0}$ is the only fixed point of $G$ in $X$ (otherwise we are done).

Furthermore, we have the following:

Lemma 2.6 The fixed point $u_{0}$ is an interior point of $X=[\underline{u}, \bar{u}]$, that is, there exists a positive number $\varepsilon$ such that $B_{E}\left(u_{0}, \varepsilon\right) \subset X$. Here

$$
B_{E}\left(u_{0}, \varepsilon\right):=\left\{v \in E:\left\|v-u_{0}\right\|_{C^{1}(\bar{\Omega})}<\varepsilon\right\}
$$

is an open ball with radius $\varepsilon$ about $u_{0}$ in $E=C_{B}^{1}(\bar{\Omega})$.

Pro of. Indeed, it suffices to note (see Lemma 2.3) that the mapping $G=K_{\omega} F_{\omega}\left(\cdot, t^{*}\right): X \rightarrow E$ is strongly increasing and that $\underline{u}<G(\underline{u})<u_{0}=G\left(u_{0}\right)<G(\bar{u})<\bar{u}$ in $X$.

Step 5: By Lemma 2.6, it follows that the Leray-Schauder fixed point index

$$
i\left(G, B_{E}\left(u_{0}, \varepsilon\right), E\right):=\operatorname{deg}\left(I-G, B_{E}\left(u_{0}, \varepsilon\right), 0\right)
$$

is well defined (cf. [2], [6], [11]). By making use of the excision property of the Leray-Schauder degree, we obtain that

$$
i(G, X, X)=\operatorname{deg}(I-G, X, 0)=\operatorname{deg}\left(I-G, B_{E}\left(u_{0}, \varepsilon\right), 0\right) .
$$

However, we have the following:

Lemma $2.7 i(G, X, X)=1$.

Proof. Since $X$ is convex and since $u_{0}$ is the only fixed point of $G$ in $X$, we have, for all $u \in \partial X$ and $0 \leq \theta \leq 1$,

$$
H(u, \theta):=(1-\theta) G(u)+\theta u_{0} \neq u .
$$

Hence, by using the homotopy invariance and normalization of the fixed point index we obtain that

$$
i(G, X, X)=\operatorname{deg}(I-H(\cdot, 0), X, 0)=\operatorname{deg}(I-H(\cdot, 1), X, 0)=i\left(u_{0}, X, X\right)=1 .
$$

This proves Lemma 2.7.

Therefore, we have proved the formula

$$
i(G, X, X)=\operatorname{deg}\left(I-G, B_{E}\left(u_{0}, \varepsilon\right), 0\right)=1 .
$$

Step 6: In order to prove that there exists another fixed point $u_{1}$ of $G$ in $X$, we make use of a generalization of Kronecker's existence theorem for the Brouwer degree (see [6, Section 3.4]):

Theorem 2.8 Let $D$ be a bounded open subset of a real Banach space $E$ and let $T: \bar{D} \rightarrow E$ be a compact mapping. If $p \notin(I-T)(\partial D)$ and if $\operatorname{deg}(I-T, D, p) \neq 0$, then there exists a point $x \in D$ such that $x-T x=p$.

The next lemma is an essential step in our proof: 
Lemma 2.9 There exists a positive number $\rho$ such that $B_{E}\left(u_{0}, \varepsilon\right) \subset B_{E}(0, \rho)$ and we have, for all $t \in I:=$ $\left[t^{*}, t_{0}+1\right]$ and all $u \in \partial B_{E}(0, \rho)$,

$$
K_{\omega}\left(F_{\omega}(u, t)\right) \neq u .
$$

Pro of. The proof of Lemma 2.9 is divided into three steps.

Step 6-1: First, we remark (see [13, Proposition 3.12]) that the resolvent $K_{\omega}: E \rightarrow E$ is strongly positive and compact. By applying the Krěn-Rutman theorem ( [13, Theorem 2.1]) to the operator $K_{\omega}$, we obtain that the spectral radius

$$
r\left(K_{\omega}\right):=\lim _{n \rightarrow \infty} \sqrt[n]{\left\|K_{\omega}^{n}\right\|}
$$

is positive and the only eigenvalue of $K_{\omega}$ having a positive eigenfunction. Hence we have the formula

$$
r\left(K_{\omega}\right)=\frac{1}{\lambda_{1}+\omega} .
$$

Indeed, it suffices to note that

$$
\begin{aligned}
\begin{cases}A \varphi_{1}=\lambda_{1} \varphi_{1} & \text { in } \Omega, \\
\varphi_{1}>0 & \text { in } \Omega, \\
B \varphi_{1}=0 & \text { on } \partial \Omega\end{cases} & \Longleftrightarrow \begin{cases}(A+\omega) \varphi_{1}=\left(\lambda_{1}+\omega\right) \varphi_{1} & \text { in } \Omega, \\
\varphi_{1}>0 & \text { in } \Omega, \\
B \varphi_{1}=0 & \text { on } \partial \Omega\end{cases} \\
& \Longleftrightarrow \begin{cases}K_{\omega} \varphi_{1}=\left(\frac{1}{\lambda_{1}+\omega}\right) \varphi_{1} & \text { in } E, \\
\varphi_{1}>0 & \text { in } \Omega .\end{cases}
\end{aligned}
$$

Moreover, it should be noticed that

$$
r(K):=\lim _{n \rightarrow \infty} \sqrt[n]{\left\|K^{n}\right\|}=\frac{1}{\lambda_{1}} .
$$

Step 6-2: By condition (F.3a) and (F.3b), we can find numbers $\mu<\lambda_{1}+\omega$ and $k \geq 0$ such that we have, for all $\xi \in \mathbf{R}$ and all $t \in I=\left[t^{*}, t_{0}+1\right], t \in I$,

$$
f_{\omega}(x, \xi, t)=f(x, \xi, t)+\omega \xi \geq \mu \xi-k \quad \text { for all } x \in \bar{\Omega}
$$

(see [14, inequalities (4.18), (4.19) and (4.20)]). If $u(x)$ is a real-valued, continuous function on $\bar{\Omega}$, it follows from an application of inequality (2.7) with $\xi:=u(x)$ that

$$
F_{\omega}(u, t) \geq \mu u-k, \quad t \in I .
$$

Now let $w \in E$ be the unique solution of the operator equation

$$
w-\mu K_{\omega} w=-k K_{\omega} 1 .
$$

The next claim asserts that the fixed points of $K_{\omega} F_{\omega}(\cdot, t)$ are uniformly bounded from below, for all $t \in I$ :

Claim 2.10 Let $u_{t}$ be an arbitrary fixed point of the mapping $K_{\omega} F_{\omega}(\cdot, t)$ with $t \in I=\left[t^{*}, t_{0}+1\right]$ :

$$
u_{t}=K_{\omega}\left(F_{\omega}\left(u_{t}, t\right)\right) .
$$

Then we have, for every $t \in I$,

$$
u_{t} \geq w .
$$


Proof. Indeed, we have, by formulas (2.10) and (2.9) and inequality (2.8) with $u:=u_{t}$,

$$
\begin{aligned}
u_{t}-w-\mu K_{\omega} u_{t}+\mu K_{\omega} w & =u_{t}-\mu K_{\omega} u_{t}-\left(w-\mu K_{\omega} w\right) \\
& =K_{\omega}\left(F_{\omega}\left(u_{t}, t\right)\right)-\mu K_{\omega} u_{t}+k K_{\omega} 1 \\
& =K_{\omega}\left(F_{\omega}\left(u_{t}, t\right)-\mu u_{t}+k\right) \\
& \geq 0 \quad \text { in } \Omega,
\end{aligned}
$$

or equivalently,

$$
\begin{cases}\left(I-\mu K_{\omega}\right)\left(u_{t}-w\right)=h & \text { in } \Omega \\ h:=K_{\omega}\left(F_{\omega}\left(u_{t}, t\right)-\mu u_{t}+k\right) \geq 0 & \text { in } \Omega\end{cases}
$$

However, since we have the inequality (see formula (2.6))

$$
r\left(\mu K_{\omega}\right)=\frac{\mu}{\lambda_{1}+\omega}<1,
$$

by applying [13, Theorem 2.2] with $\lambda:=1$ and $K:=\mu K_{\omega}$ we obtain that equation (2.12) has the only positive solution $u_{t}-w$ for $h \geq 0$ in the ordered Banach space $E=C_{B}^{1}(\bar{\Omega})$. Therefore, we have the desired assertion

$$
u_{t}(x) \geq w(x) \quad \text { in } \Omega .
$$

The proof of Claim 2.10 is complete.

Step 6-3: In order to prove Lemma 2.9, we assume, to the contrary, that assertion (2.5) does not hold true. Then we can find a sequence $\left\{t_{j}\right\} \subset I=\left[t^{*}, t_{0}+1\right]$ and a sequence $\left\{u_{j}\right\} \subset E=C_{B}^{1}(\bar{\Omega})$ such that

$$
\left\|u_{j}\right\|_{E} \longrightarrow \infty \quad \text { as } j \rightarrow \infty
$$

and that

$$
u_{j}=K_{\omega}\left(F_{\omega}\left(u_{j}, t_{j}\right)\right) .
$$

We let

$$
v_{j}(x):=\frac{u_{j}(x)}{\left\|u_{j}\right\|_{E}} \quad \text { for all } x \in \bar{\Omega},
$$

and divide the both sides of formula (2.14) by $\left\|u_{j}\right\|_{E}$ to obtain that

$$
v_{j}=\frac{u_{j}}{\left\|u_{j}\right\|_{E}}=K_{\omega}\left(\frac{F_{\omega}\left(u_{j}, t_{j}\right)}{\left\|u_{j}\right\|_{E}}\right) .
$$

However, by using condition (F.4) and inequality (2.11) we find that the sequence

$$
\frac{F_{\omega}\left(u_{j}, t_{j}\right)}{\left\|u_{j}\right\|_{E}}
$$

is bounded in the space $C(\bar{\Omega})$. Since the mapping $K_{\omega}: C(\bar{\Omega}) \rightarrow C^{1}(\bar{\Omega})$ is compact, it follows from formula (2.15) that the sequence $\left\{v_{j}\right\}$ contains a convergent subsequence in the space $C^{1}(\bar{\Omega})$. Hence, by passing to an appropriate subsequence we may assume that

$$
\left\{\begin{array}{l}
v_{j} \longrightarrow v \quad \text { in } E=C_{B}^{1}(\bar{\Omega}) \text { as } j \rightarrow \infty, \\
\|v\|_{E}=1 .
\end{array}\right.
$$


Indeed, it suffices to note that

$$
B v=\lim _{j \rightarrow \infty} B v_{j}=0 \quad \text { on } \partial \Omega
$$

and further that

$$
\|v\|_{E}=\lim _{j \rightarrow \infty}\left\|v_{j}\right\|_{E}=1
$$

Moreover, by formula (2.15) and inequality (2.11) it follows that

$$
v_{j}=\frac{u_{j}}{\left\|u_{j}\right\|_{E}} \geq \frac{w}{\left\|u_{j}\right\|_{E}} .
$$

By passing to the limit in inequality (2.17), we obtain from conditions (2.13) and (2.16) that

$$
v \geq 0 \quad \text { in } E \text {. }
$$

On the other hand, by condition (F.3b) we can find numbers $\gamma>0$ and $\delta \geq 0$ such that, for all $\xi \geq 0$ and all $t \in I$

$$
f_{\omega}(x, \xi, t)=f(x, \xi, t)+\omega \xi \geq\left(\lambda_{1}+\omega+\gamma\right) \xi-\delta \quad \text { for all } x \in \bar{\Omega}
$$

(see [14, inequalities (4.19) and (4.20)]). By inequality (2.19) with $\xi:=u_{j}(x)$ and $t:=t_{j}$, it follows that

$$
F_{\omega}\left(u_{j}, t_{j}\right) \geq\left(\lambda_{1}+\omega+\gamma\right) u_{j}-\delta
$$

so that

$$
\frac{F_{\omega}\left(u_{j}, t_{j}\right)}{\left\|u_{j}\right\|_{E}} \geq\left(\lambda_{1}+\omega+\gamma\right) \frac{u_{j}}{\left\|u_{j}\right\|_{E}}-\frac{\delta}{\left\|u_{j}\right\|_{E}}=\left(\lambda_{1}+\omega+\gamma\right) v_{j}-\frac{\delta}{\left\|u_{j}\right\|_{E}} .
$$

Hence we have, by formula (2.15) and the positivity of $K_{\omega}$,

$$
v_{j}=\frac{K_{\omega}\left(F_{\omega}\left(u_{j}, t_{j}\right)\right)}{\left\|u_{j}\right\|_{E}} \geq\left(\lambda_{1}+\omega+\gamma\right) K_{\omega} v_{j}-\frac{\delta K_{\omega} 1}{\left\|u_{j}\right\|_{E}} .
$$

Therefore, by passing to the limit in inequality (2.20) we obtain from conditions (2.16) and (2.13) that

$$
v \geq\left(\lambda_{1}+\omega+\gamma\right) K_{\omega} v
$$

or equivalently,

$$
\begin{cases}\left(I-\left(\lambda_{1}+\omega+\gamma\right) K_{\omega}\right) v=g & \text { in } \Omega \\ g \geq 0 & \text { in } \Omega .\end{cases}
$$

However, since we have the inequality (see formula (2.6))

$$
r\left(\left(\lambda_{1}+\omega+\gamma\right) K_{\omega}\right)=\frac{\lambda_{1}+\omega+\gamma}{\lambda_{1}+\omega}>1
$$

by applying [13, Theorem 2.2] with $\lambda:=1$ and $K:=\left(\lambda_{1}+\omega+\gamma\right) K_{\omega}$ we obtain that equation (2.21) has no positive solution for any given $g \geq 0$ in the ordered Banach space $E$. Hence it follows from assertion (2.18) that

$$
v=0 \text { in } E \text {. }
$$

This contradicts condition (2.16) that $\|v\|_{E}=1$.

The proof of Lemma 2.9 is complete. 
Step 7: By Lemma 2.9, we can apply the homotopy invariance of the Leray-Schauder degree to obtain that

$$
\begin{aligned}
\operatorname{deg}\left(I-G, B_{E}(0, \rho), 0\right) & =\operatorname{deg}\left(I-K_{\omega} F_{\omega}\left(\cdot, t^{*}\right), B_{E}(0, \rho), 0\right) \\
& =\operatorname{deg}\left(I-K_{\omega} F_{\omega}\left(\cdot, t_{0}+1\right), B_{E}(0, \rho), 0\right) \\
& =0,
\end{aligned}
$$

since the operator $K_{\omega} F_{\omega}\left(\cdot, t_{0}+1\right)$ has no fixed point in $E$.

Therefore, by the domain additivity of the Leray-Schauder degree it follows from formulas (2.4) and (2.22) that

$$
\begin{aligned}
\operatorname{deg}\left(I-G, B_{E}(0, \rho) \backslash \overline{B_{E}\left(u_{0}, \varepsilon\right)}, 0\right) & =\operatorname{deg}\left(I-G, B_{E}(0, \rho), 0\right)-\operatorname{deg}\left(I-G, B_{E}\left(u_{0}, \varepsilon\right), 0\right) \\
& =-1
\end{aligned}
$$

By applying Kronecker's existence theorem for the Leray-Schauder degree (Theorem 2.8) with

$$
E:=C_{B}^{1}(\bar{\Omega}), \quad D:=B_{E}(0, \rho) \backslash \overline{B_{E}\left(u_{0}, \varepsilon\right)}, \quad T:=G, \quad p:=0,
$$

we can find a fixed point $u_{1}$ of $G$ in $B_{E}(0, \rho) \backslash \overline{B_{E}\left(u_{0}, \varepsilon\right)}$. Namely, we have the equivalent assertions

$$
\begin{aligned}
u_{1}=G u_{1} \text { in } E & \Longleftrightarrow u_{1}=K_{\omega}\left(F_{\omega}\left(u_{1}, t^{*}\right)\right) \text { in } E \\
& \Longleftrightarrow \begin{cases}(A+\omega) u_{1}=f_{\omega}\left(x, u_{1}(x), t^{*}\right) & \text { in } \Omega, \\
B u_{1}=0 & \text { on } \partial \Omega\end{cases} \\
& \Longleftrightarrow \begin{cases}A u_{1}=f\left(x, u_{1}(x), t^{*}\right) & \text { in } \Omega, \\
B u_{1}=0 & \text { on } \partial \Omega .\end{cases}
\end{aligned}
$$

Summing up, we have proved that problem (1.3) has at least two distinct solutions $u_{0}, u_{1}$ for $t=t^{*}$.

Step 8: Finally, in order to prove that problem (1.3) has a solution also for $t=t_{0}$, we take an increasing sequence $\left\{t_{j}\right\}$ such that $t_{j} \uparrow t_{0}$. Then, by arguing just as in Step 6-3 we find that the corresponding solutions

$$
u_{j}=K_{\omega}\left(F_{\omega}\left(u_{j}, t_{j}\right)\right)
$$

remain bounded in $E$. Moreover, by Lemma 2.2 we may assume that the sequence $\left\{u_{j}\right\}$ itself converges to some function $u$ in $E$ as $j \rightarrow \infty$. Therefore, by passing to the limit in formula (2.23) we obtain that

$$
u=K_{\omega}\left(F_{\omega}\left(u, t_{0}\right)\right) \text { in } E=C_{B}^{1}(\bar{\Omega}) .
$$

This proves that $u \in W^{2, p}(\Omega)$, with $N<p<\infty$, is a solution of problem (1.3) for $t=t_{0}$. Indeed, it suffices to note that

$$
u=K_{\omega}\left(F_{\omega}\left(u, t_{0}\right)\right) \text { in } E \Longleftrightarrow \begin{cases}A u=f\left(x, u(x), t_{0}\right) & \text { in } \Omega, \\ B u=0 & \text { on } \partial \Omega .\end{cases}
$$

Now the proof of Theorem 1.1 is complete.

Acknowledgements The author is grateful to one of the three referees of the previous paper [14] who suggested studying problem (1.3) in the critical case where $t=t_{0}$. Furthermore, the author would like to thank the two referees of the present paper for their helpful suggestions from the viewpoint of nonlinear analysis. This research is partially supported by Grantin-Aid for General Scientific Research (No. 19540162), Ministry of Education, Culture, Sports, Science and Technology, Japan. 


\section{References}

[1] R.A. Adams and J.J.F. Fournier, Sobolev spaces, second edition (Academic Press, Amsterdam Heidelberg New York Oxford, 2003).

[2] H. Amann, Fixed point equations and nonlinear eigenvalue problems in ordered Banach spaces, SIAM Rev. 18 (1976), 620-709.

[3] H. Amann and P. Hess, A multiplicity result for a class of elliptic boundary value problems, Proc. Roy. Soc. Edinburgh 84A (1979), 145-151.

[4] A. Ambrosetti and G. Prodi, On the inversion of some differentiable mappings with singularities between Banach spaces, Ann. Mat. Pura Appl. 93 (1972), 231-247.

[5] M.S. Berger and E. Podolak, On the solutions of a nonlinear Dirichlet problem, Indiana Univ. Math. J. 24 (1975), $837-846$

[6] K.C. Chang, Methods in nonlinear analysis (Springer-Verlag, Berlin Heidelberg New York, 2005).

[7] L. Hörmander, The analysis of linear partial differential operators III (Springer-Verlag, Berlin Heidelberg New York Tokyo, 1985).

[8] J.L. Kazdan and F.W. Warner, Remarks on some quasilinear elliptic equations, Comm. Pure Appl. Math. 28 (1975), 567-597.

[9] J. Leray et J. Schauder, Topologie et équations fonctionelles, Ann. Sci. École Norm. Sup. 51 (1934), 45-78.

[10] J. Mawhin, Topological degree methods in nonlinear boundary value problems, CBMS Regional Conference Series in Mathematics, no. 40 (American Mathematical Society, Providence, Rhode Island, 1979).

[11] L. Nirenberg, Topics in nonlinear functional analysis (Courant Institute of Mathematical Sciences, New York University, New York, 1974).

[12] T. Runst and W. Sickel, Sobolev spaces of fractional order, Nemytskij operators, and nonlinear partial differential equations (Walter de Gruyter, Berlin New York, 1996).

[13] K. Taira, Degenerate elliptic boundary value problems with indefinite weights, Mediterranean J. Math. 5 (2008), 133162.

[14] K. Taira, Multiple solutions of semilinear degenerate elliptic boundary value problems, Math. Nachr.

[15] E. Zeidler, Nonlinear functional analysis and its applications I (Springer-Verlag, New York Berlin Heidelberg, 1986). 